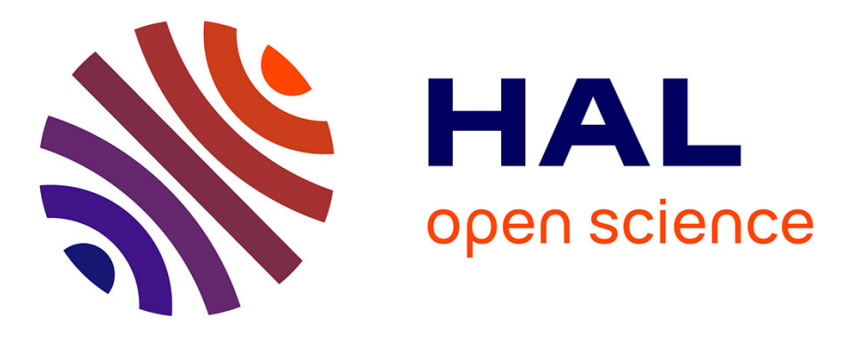

\title{
Use of ambiguous detections to improve estimates from species distribution models
}

Julie Louvrier, Anja Molinari-jobin, Marc Kéry, Thierry Chambert, David Miller, Fridolin Zimmermann, Eric Marboutin, Paolo Molinari, Oliver Müeller, Rok Černe, et al.

\section{To cite this version:}

Julie Louvrier, Anja Molinari-jobin, Marc Kéry, Thierry Chambert, David Miller, et al.. Use of ambiguous detections to improve estimates from species distribution models. Conservation Biology, 2018, 33, pp.185 - 195. 10.1111/cobi.13191 . hal-03502432

\section{HAL Id: hal-03502432 \\ https://hal.science/hal-03502432}

Submitted on 4 Jan 2022

HAL is a multi-disciplinary open access archive for the deposit and dissemination of scientific research documents, whether they are published or not. The documents may come from teaching and research institutions in France or abroad, or from public or private research centers.
L'archive ouverte pluridisciplinaire HAL, est destinée au dépôt et à la diffusion de documents scientifiques de niveau recherche, publiés ou non, émanant des établissements d'enseignement et de recherche français ou étrangers, des laboratoires publics ou privés. 
Use of ambiguous detections to improve estimates from species distribution models.

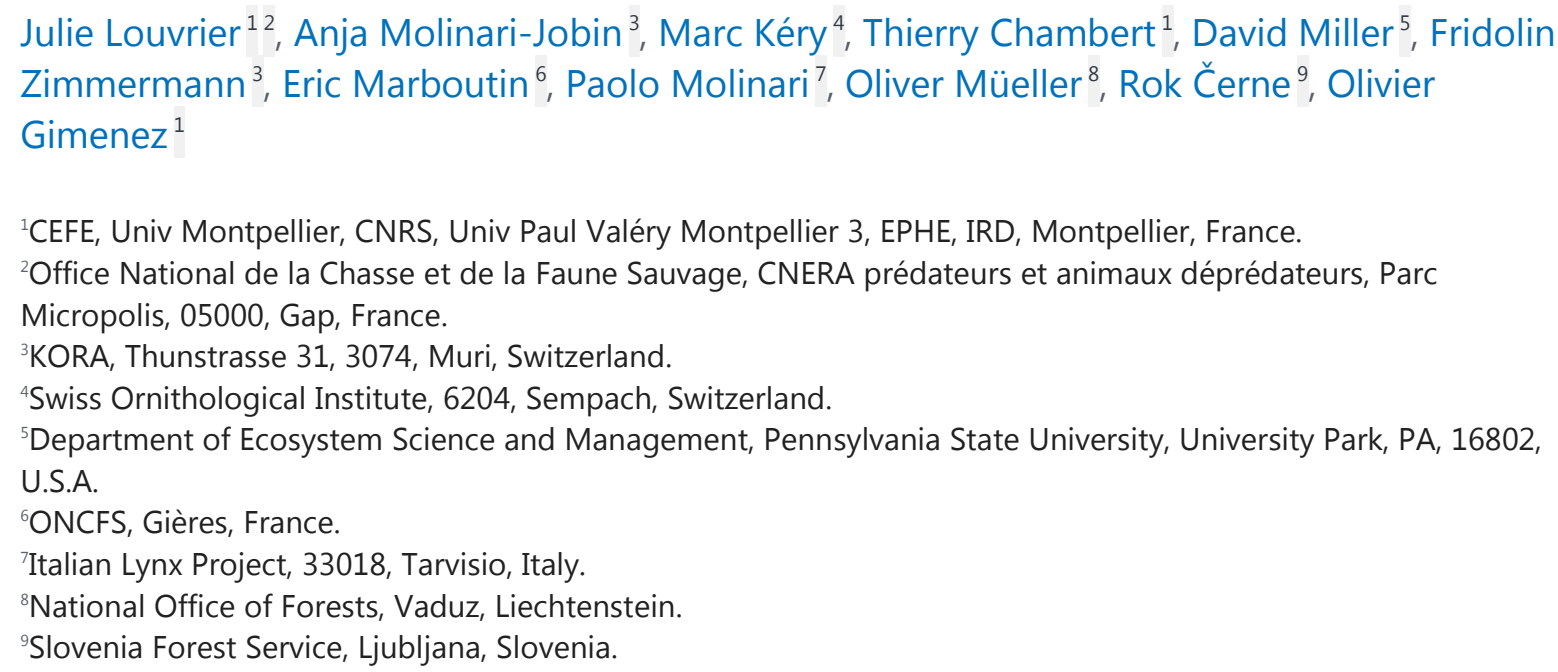

\section{Abstract}

As large carnivores recover throughout Europe, there is a need to study their distribution to determine their conservation status and assess the potential for conflicts with human activities. However, efficient monitoring of many large carnivore species is challenging due to their rarity, elusive behavior and large home range size. In Europe, most current monitoring protocols rely on multiple detection methods, which can include opportunistic sightings from citizens in addition to designed surveys. Two types of detection errors may occur in such monitoring schemes; false negatives and false positives. When not accounted for, both can bias estimates from species distribution models (SDMs). False negative detections can be accounted for in SDMs that deal with imperfect detection. In contrast, false positive detections, due to species misidentification, have only rarely been accounted for in SDMs. Generally, researchers use ad hoc methods to avoid false positives through data filtering to discard ambiguous observations prior to analysis. These practices may discard valuable ecological information on the distribution of a species. Here, we investigated the costs and benefits of including data types that might include false positives rather than discard them for SDMs of large carnivores. We showcase a dynamic occupancy model that simultaneously 
accounts for false negatives and positives to jointly analyze data that include both unambiguous detections and ambiguous detections. Using simulations, we show that the addition of ambiguous detections increases the precision of parameter estimates. The analysis of data on the Eurasian lynx (Lynx lynx) suggested that incorporating ambiguous detections produced more precise estimates of the ecological parameters and revealed additional occupied sites in areas where the species is likely expanding. Overall, our work shows that ambiguous data should be considered when studying the distribution of large carnivores, through the use of dynamic occupancy models accounting for misidentification.

\section{Introduction}

The distribution and abundance of large carnivores in many parts of the world has been declining for centuries because of habitat loss and human persecution (Ripple et al. 2014). Thanks to active conservation measures, several species of large carnivores have recently been expanding their ranges substantially in Europe. As a result, most European countries currently host at least one viable population of large predators (Chapron et al. 2014). This recent expansion led to the emergence of conflicts with humans (Ripple et al. 2014). In this context, accurate distribution mapping, i.e., species distribution models (SDMs; Elith \& Leathwick 2009), is essential to determine the conservation status and recovery success (IUCN, 2012), to target potential areas of occurrence and understand large carnivores range dynamics, identify the possible areas where they might be recovering in the future (Chapron et al., 2014) and mitigate conflicts often associated with the recovery of large carnivores (Guillera-Arroita et al. 2015) like, e.g., livestock depredation related to wolves' recolonization (Marucco \& Mcintire, 2010). However, their rarity, elusive behavior and low density render efficient monitoring of large carnivores difficult (Ripple et al. 2014). 
The monitoring of large carnivores in Europe relies on several survey methods that are implemented by professionals and members of the public ("citizens"). Citizens in particular add to the ability to survey large spatial coverage over extended periods in time, which would be costly if done by professionals only (Molinari-Jobin et al. 2017). The practice of engaging the public in a project that produces reliable data and information usable by scientists and/or decision-makers is a primary goal of citizen science (CS; McKinley et al. 2017). CS is becoming an important tool in ecology to study the distribution, abundance and species richness of plants and animals (Silvertown 2009; Dickinson et al. 2012). However, CSgenerated data present potential quality issues especially when the goal is to build SDMs.

Difficulty detecting large carnivores means that animals can be missed at sites where they are present (i.e., producing false negative observations). Occupancy models were developed to deal with false negative errors (Guillera-Arroita 2017) and are recommended for analyzing CS data (Isaac et al. 2014). While datasets produced by CS have been proven valuable (Kosmala et al. 2016), professionals may present a better expertise than citizens to detect or identify the species of interest, diminishing the risk of identification errors (Fitzpatrick et al. 2009). False positives can occur when the species of interest is "detected" at a site where it does not occur, resulting from misidentification (Miller et al. 2011). Recent studies have demonstrated the importance of accounting for misidentification for SDMs (Miller et al. 2011, 2013; Chambert et al. 2015). Ignoring misidentification may lead to overestimating species range (Royle \& Link 2006; McClintock et al. 2010).

Methods of observations typically used to survey large carnivores are based on indirect observation methods through signs of presence such as tracks, prey remains, cameratrap photos, or scats (Molinari-Jobin et al. 2017). Observations then go through a filtering process performed by experts to assess the reliability of evidence of presence. Recent studies of the distribution of European large carnivores were based only on the reliable observations, 
i.e., those remaining after discarding ambiguous detections and validated by experts (Molinari-Jobin et al. 2017). This means that part of the observations may end up being discarded, even though they may contain relevant ecological information on the species distribution. This raises the question whether this information can somehow be extracted and made useful in the context of SDMs?

Here, we investigated the pros and cons of removing ambiguous detections in SDMs of large carnivores versus keeping all records and formally accounting for misidentification. We showcase a dynamic occupancy model accounting for both false negative and false positive errors (Miller et al. 2011, 2013) to jointly analyze unambiguous and ambiguous detections. To assess the performance of this approach, we performed a simulation study which compares the analysis of unambiguous and ambiguous detections vs. using unambiguous detections only.

We illustrate these methods in a case study with a SDM of the Eurasian lynx (Lynx lynx) throughout the European Alps (Molinari-Jobin et al. 2017). Observations differ in their reliability in terms of the likely incidence of false positives. Ambiguous detections, which are usually discarded, represent almost a third of all observations in the dataset and have a larger geographic range than unambiguous detections. We expected improved precision in ecological parameter estimates when all data were included in an analysis, despite having to accommodate additional nuisance parameters to deal with misidentification.

\section{Material and Methods}

\section{1- Occupancy model accounting for misidentification}

Dynamic occupancy models allow the estimation of occupancy and its temporal dynamics as a function of local extinction and colonization probabilities, while accounting for imperfect 
species detection (MacKenzie et al. 2003). These models can be formulated as state-space models to separate the state process, whether a species is present or not at a site and how that changes through time, from the observational process, whether the species is observed at a site during a given period depending on whether or not it was actually present (Royle \& Kéry 2007). We define $z_{i, 1}$ as the initial latent occurrence state of site $i$ (with $\mathrm{z}=1$ denoting presence and $\mathrm{z}=0$ absence), and $\mathrm{z}_{i, t}$ the latent state for of site $i$ at time $t$. The state process is initiated by the initial occupancy probability $\psi_{i, 1}$ for site $i$, then governed by colonization probability $\gamma_{i, t}$ (the probability that a site $i$ that is not occupied at time $t$ will become occupied at time $t+1$ ), and extinction probability $\varepsilon_{i, t}$ (the probability that an occupied site $i$ at time $t$ will become unoccupied at time $t+1$ ). We model $z_{i, 1}$ as a draw from a Bernoulli distribution with probability $\psi_{i, 1}$. All subsequent latent states $z_{i, t}$ for $t>1$ are draws from another Bernoulli distribution that combines both possible extinction and colonization events:

$z_{i, t+1} \mid z_{i, t} \sim \operatorname{Bernoulli}\left(z_{i, t}\left(1-\varepsilon_{i, t}\right)+\left(1-z_{i, t}\right) \gamma_{i, t}\right)$.

If a site is occupied in year (or season) $t$ it will still be occupied with probability $1-\varepsilon_{i, t}$ or if it is unoccupied it will become occupied with probability $\gamma_{i, t}$. Each site is surveyed during secondary occasions (or survey) $j$ within year (or season) $t$. Site occupancy models rely satisfying the site closure assumption, whereby the latent occurrence state of a site does not change within a sampling season, whereas occupancy dynamics (colonization, extinction) happen between years (or seasons).

In addition to the state process, the observation process leads to the data $y_{i, j, t}:$ the observed state of site $i$ during secondary occasion (or survey) $j$ within year (or season) $t$. Hereafter, we drop the indices when possible to ease the reading. In our study, $y=0$ denoted no detection, $y=1$ an unambiguous detection and $y=2$ an ambiguous detection. To account for unambiguous and ambiguous detections, we followed the formulation of Miller et al. 
(2013). We defined an additional parameter $d_{i, j, t}$ which took the value of 1 if any detection (ambiguous or unambiguous) was made at site $i$ during survey $j$ within year $t$, and 0 if not. For occupied sites, by definition $d=1$ denoted a true detection while for unoccupied sites, $d=1$ denoted a false positive detection. For both occupied and unoccupied sites, $d=0$ meant no detection was made hence, $y=0$. At an occupied site, the possible observations are: no detection $(y=0)$, unambiguous detection $(y=1 \mid d=1)$ or ambiguous detection $(y=2 \mid d=1)$. For occupied sites, the probability of a true detection (i.e. $d=1$ ) during a secondary sampling occasion (or survey) is defined as $P(d=1 \mid z=1)$, hereafter written as $p_{11}$. The probability that a true detection will be classified as unambiguous is given by the probability $P(y=1 \mid d=1)$ hereafter written as $b$. The probability of an unambiguous detection is $p_{11} b$ and the probability for an ambiguous detection (i.e. $y=2)$ for an occupied site is $p_{11}(1-b)$. For unoccupied sites (i.e. $z=0)$, by definition, unambiguous detections $(y=1 \mid d=1)$ do not occur thus, only two possible observations can be made: an ambiguous detection $(y=2 \mid d=1)$, which in this case is a false positive, or no detection $(y=0)$. The probability of a false positive detection (i.e. $d=$ 1) occurring at an unoccupied site $i$ during a secondary sampling occasion (or survey) $j$ is $P(d=1 \mid z=0)$, hereafter written as $p_{10}$. Then the probabilities, unconditional on state $\mathrm{z}$ of a site, of recording the three possible observed states $(y)$ are:

$\mathrm{P}(y=0)=P(z=1) P(d=0 \mid z=1)+P(z=0) P(d=0 \mid z=0)$

$$
=\psi\left(1-p_{11}\right)+(1-\psi)\left(1-p_{10}\right) \text { for no detection; }
$$

$\mathrm{P}(y=1)=P(z=1) P(d=1 \mid z=1) P(y=1 \mid d=1)$

$=\psi p_{11} b$ for unambiguous detection;

$\mathrm{P}(y=2)=P(z=1) P(d=1 \mid z=1) P(y=2 \mid d=1)+P(z=0) P(d=1 \mid z=0)$

$$
=\psi p_{11}(1-b)+(1-\psi) p_{10} \text { for ambiguous detection. }
$$


We conducted a simulation study to examine the performance of a dynamic occupancy model that also accounts for possible false positives (MUA - "Model Unambiguous/Ambiguous") in comparison with the dynamic occupancy model that only accounts for false-negatives, i.e. fitted with unambiguous data only (MU - "Model Unambiguous"). To assess the ability of both models to estimate ecological parameters, we defined four scenarios in which parameters which control false positive detections and true detections varied (Table 1).

First, because the ecological parameters have an influence on the amount of detections produced, we chose two main situations in which the occupancy probability is either "high" or "low". In the "high" occupancy scenario, we set the initial occupancy probability $\psi_{1}$ at 0.8 , the colonization probability $\gamma$ at 0.4 and extinction probability at 0.1 to maintain a high occupancy probability. This scenario would correspond to a fairly well-established species reflected by its high occupancy probability across time. In the "low" occupancy scenario, we set the initial occupancy probability $\psi_{1}$ at 0.1 , the colonization probability $\gamma$ at 0.1 and extinction probability at 0.1 to maintain a low occupancy probability. This scenario would correspond to a rare species with a low occupancy probability across time.

172 The detection parameters also have an influence on the amount of false positive and true 173 positive detections. First, true detections are controlled by the true detection probability $p_{11}$ and the probability to classify a true detection as unambiguous $b$. Therefore, in both "high" and "low" occupancy scenarios, we consider two situations in which $b$ is either "high" (i.e. set at 0.8 ) or "low" (i.e. set at 0.5 ), leading to four scenarios. For all scenarios, we set $p_{11}$ at 0.4 .

177 When $b$ is equal to 0.8 , most of the true detections are classified as unambiguous. This 
scenario would correspond to the monitoring of a species that is not easily mistaken for another one or done by people trained to recognize accurately the presence signs of the species. When $b$ is equal to 0.5 , a larger part of the true detections is classified as ambiguous. This scenario would correspond to the monitoring of a species that can easily be mistaken or done by untrained people, for instance from the general public. Second, the amount of false positive detections is controlled by the false positive detection probability $p_{10}$. In all four scenarios, we looked at how the models performed under seven different values of $p_{10}$, varying from 0.01 to 0.3 , leading to twenty-eight different simulation scenarios. Finally, because our main objective was to assess the effect of accounting for ambiguous data, environmental variation was not included into our simulation study.

For ease of reading, the "high" occupancy "high" $b$ scenario will be referred to as $\mathrm{HH}$; the "high" occupancy "low" $b$ will be HL; the "low" occupancy "high" $b$ will be LH; and the "low" occupancy "low" $b$ will be LL.

In our simulations, we generated data for 100 sites over 5 years and 3 surveys. To remain realistic in the simulations, the number of surveys were chosen to mimic the case study characteristics. For each scenario, we simulated $S=500$ datasets and we fitted both models to each dataset. For the initial occupancy probability $\psi_{1}$, the colonization probability $\gamma$ and the extinction probability $\varepsilon$ in both models in each scenario, we calculated the relative bias and mean squared error (MSE).

\section{3- Case study: Eurasian lynx in the Alps 1995-2014}

After its total eradication in the Alps by around 1930, the Eurasian lynx (Lynx lynx) has been reintroduced multiple times between 1970 and today in Switzerland, Italy, Austria and Slovenia (Molinari-Jobin et al. 2017). In the 1990s, experts from the seven Alpine countries 
set up the international lynx monitoring program SCALP (Status and Conservation of the Alpine Lynx Population). The monitoring of the elusive lynx, relies on a network of $>1300$ trained experts (game wardens, hunters, and naturalists) covering seven Alpine countries. Signs of presence were classified into three reliability categories: C1 included "hard facts" data, e.g. dead lynx, lynx removed from the wild as young orphans and put into captivity, lynx photos and a few genetic samples, C2 are detections that were confirmed by a lynx expert, (all livestock killed by lynx that was compensated, verified wild prey remains, and tracks) and C3 are data that could not be verified by experts (unverified tracks and wild prey remains) and unverifiable data such as any sighting, scats and vocalizations. We treated $\mathrm{C} 1$ and $\mathrm{C} 2$ data as unambiguous detections, assuming there were no false-positive detections in these data, while the C3 data were treated as ambiguous detections. From 1995 to 2014,8415 observations (67\%) were classified as unambiguous detections and 3991 (33\%) as ambiguous. If unambiguous and ambiguous detections occurred at a site, we accounted for the unambiguous detections only. Non-detections were obtained on the sites that were sampled but where no lynx presence was reported during a survey within a year. In Molinari-Jobin et al. (2017), a dynamic occupancy model was fitted using unambiguous detections only (i.e., using our model MU) to assess the effects of environmental covariates on different parameters of the model and to assess distribution-based population trends. A $10 \times 10 \mathrm{~km}$ grid was used to define the distribution units which correspond to the approximate size of female lynx' home-range in the Alps (Molinari-Jobin et al. 2017). Surveys were defined as three replicated two-month periods: November-December; January-February; and March-April. Here, we used the same data set as did Molinari-Jobin et al. (2017), but in addition we also used the $\mathrm{C} 3$ data and fitted a dynamic occupancy model that combined both unambiguous and ambiguous detections (MUA). In addition, we used the same covariates for the parameters that are in common in the models MU and MUA. We considered the effects of forest cover 
and distance to the release site on $\psi_{1}$; the effects of year, forest cover, and number of observed occupied contiguous neighbors on $\varepsilon$; and the same effects plus that of human density and elevation on $\gamma$.

For the new parameters in MUA, $p_{11}$ and $p_{10}$, we used the effect of elevation and forest cover and a random site-by-winter effect to accommodate unmodeled spatial heterogeneity in detection rates in every combination of site and winter. A "network" covariate was also included to account for heterogeneity in sampling effort in time and space. This covariate took the following values based on the amount of effort for the location and time period -0 : no information was available regarding the sampling effort in which case we assumed that it was small but never exactly null, owing to the large number of observers and organizations that collaborate in the Alpine lynx monitoring (Molinari-Jobin et al., 2012); 1: trained lynx monitoring network were present on the site; and 2: experienced lynx monitoring network members were actively searching for lynx signs. We also considered a linear year effect, i.e., an annual trend, on $p_{10}$ to investigate whether this probability decreased as observers gained experience over time. Finally, we kept the probability $b$ to classify a true positive detection as unambiguous constant. We considered the effect of a covariate as "significant" if its 95\% credible interval (CRI) did not overlap 0.

To evaluate the added value of incorporating the $\mathrm{C} 3$ data (ambiguous detections) into the analysis, we compared the maps of occupancy produced by the two models by calculating and mapping the difference in the site- and year-specific estimates of realized occurrence $\hat{z}_{\mathrm{i}, \mathrm{t}}$ $(M U)-\hat{z}_{\mathrm{i}, \mathrm{t}}(M U A)$

We provide the codes to run the simulations and fit the models described above in Appendix S1 and Appendix S2. 


\section{Results}

252

253

254

255

256

257

1- Simulations

When looking at the MSE, MUA performed better than MU in all 4 scenarios when the probability of false positive detection $p_{10}$ was below or equal to 0.15 (Appendix S3). Above this value of $p_{10}$ both models performed equally well except in one scenario and for one parameter when estimating the ecological parameters: MUA estimated the colonization probability $\gamma$ less precisely than MU only in the HL scenario for values of $p_{10}$ between 0.20 and 0.30. MSE was at its highest value, varying between 0.04 and 0.25 in the HL scenario, then between 0.04 and 0.14 in the HH scenario. MSE was at its lowest value in the LH scenario, varying between 0.02 and 0.06 , then between 0.02 and 0.11 in the LL scenario.

Both models estimated the initial occupancy probability $\psi_{1}$ and $\gamma$ with biases below or equal to $5 \%$ in the three scenarios HH, HL, LH (Appendix S3). In the LL scenario, MU estimated $\psi_{1}$ with a bias above 5\% (up to 8\%) and MUA had a lower bias than MU. Finally, for the extinction probability $\varepsilon$, MUA performed better or equivalently above $5 \%$ in terms of bias in the scenarios $\mathrm{HH}$ and HL, and worse or equivalently above $5 \%$ in the LH and LL scenarios.

\section{2- Lynx case study}

When we fitted the MUA with both unambiguous and ambiguous detections (i.e., for C1, $\mathrm{C} 2$ and $\mathrm{C} 3$ data), the true detection probability, $p_{11}$, was higher on sites with a high forest cover, and appeared to vary according to the season and network (Table 2). Elevation had no effect on $p_{11}$. The false positive detection probability, $p_{10}$, was higher on sites with a high forest cover and varied according to network (Table 2). While elevation and season had no significant effect on $p_{10}$, we found that this probability decreased with time (Table 2). Both 
models gave similar estimates for $\psi_{1}, \varepsilon$ and $\gamma$ but MUA produced more precise estimates than MU (Appendix S4).

The probability $b$ of classifying a true detection as unambiguous was estimated at 0.81 with high precision (CRI $0.79-0.83$ ). At the beginning of the study period, in the winter 1995/1996, we estimated the mean occupancy probability $\psi$ over all sites at 0.04 (CRI 0.03 $0.07), p_{11}$ was estimated on average at $0.11(\mathrm{CRI} 0.10-0.25)$ and $p_{10}$ was estimated at 0.006 (CRI 0.004- 0.01). For the end of the study period, the winter 2013/2014, we estimated the mean $\psi$ at $0.1(0.0899 ; 0.11), p_{11}$ was estimated on average at $0.17(0.09 ; 0.24)$ and $p_{10}$ at 0.007 (0.003; 0.010). MUA estimated a few more occupied sites than MU for both winters 1995/1996 and 2013/2014 (between 4 in 1995/1996 to 13 in 2013/2014, see Fig. 1, middle and bottom panel) and estimated occupied sites that were estimated occupied by MU too. The additional sites that were estimated occupied from MUA were sites where ambiguous detections had occurred (Fig. 1, top panel).

[Table 2 about here]

[Figure 1 about here]

\section{Discussion}

Assessing the distribution of large carnivores at large scales is a central information for assessing their conservation status, and abundance (IUCN, 2012; Jedrzejewski et al., 2018), target potential conflict areas (Marucco \& Mcintire, 2010) and understand the mechanism of the distribution's dynamics for successful management (Eriksson \& Dalerum, 2018). Producing more precise and less biased estimates by adding ambiguous data with a model accounting for false positive detections can bring new insights into species' distribution in places where getting unambiguous data is challenging. Due to the large areas involved, the 
monitoring of large carnivores in Europe relies on a large network of both professional and non-professional observers (Louvrier et al. 2018; Molinari-Jobin et al. 2017). While falsenegative detections have received much attention in the species distribution modeling literature with the rise of occupancy models (MacKenzie et al. 2003; Bailey et al. 2014), dealing with ambiguous detections has been studied much less (Miller et al. 2011; Chambert et al. 2015). Here, using simulations we demonstrate that jointly analyzing unambiguous and ambiguous detections with the appropriate dynamic occupancy models led to increased precision in the estimates of ecological parameters when $p_{10}$ was low. When this probability was above 0.20 , both models estimated ecological parameters with almost equivalent precision which varied between its highest values in the "high" occupancy scenarios and its lowest values in the "low" occupancy scenarios. Both models produced estimates of ecological parameters with low bias except for one ecological parameter in one specific scenario.

When looking at the results of the lynx analysis, we found that adding ambiguous data helped produce more precise estimates and provided additional spatial information that improved inference in areas where the species likely occurred at very low density (e.g., at a colonization front).

\section{What did we learn from the simulation study?}

MUA performed better than MU in most of the scenarios. Two factors seemed to have an influence on models' performances: the false-positive probability $p_{10}$ and the occupancy probability. In terms of precision, MUA performed better when $p_{10}$ was low and performed equivalently when $p_{10}$ was high. In the case of a low occupancy probability, the estimates of extinction probability were found to be more biased positively under the MUA than the MU 
leading to an overestimation of $\varepsilon$ and the distribution. For the other parameters and the other scenarios, MUA produced estimates with low biases. Whether a species is occurring at "high" or "low" occupancy probability can often be evaluated prior to the analyses based on the knowledge of the species ecology or on previous studies. Overall, we recommend always including ambiguous data, as in most of the scenarios MUA performed better than or equivalently to MU in terms of both precision and bias for the ecological parameter estimates.

\section{Shall we account for ambiguous data when studying the distribution of large carnivores?}

Using a model incorporating both unambiguous and ambiguous data, we estimated the effect of several covariates on the dynamics of Lynx occupancy in the entire range of the Alps. This SDM exercise allowed assessing trends in the distribution of the species, informing its conservation status (Guisan \& Thuiller 2005). We found covariate effects to be similar in direction and magnitude to those estimated by Molinari-Jobin et al. (2017) who fitted the simpler MU to the lynx data with unambiguous detections only (Table 2). We refer the reader

to their study for a detailed description of these effects and their possible biological interpretation. Our results showed that the probability to make a false positive detection decreased over time. This could be due to observers remaining in the network becoming less likely to make false positive detections with time as they became more experienced in recognizing the species (Jordan et al. 2012). This was corroborated by the fact that the number of ambiguous detections decreased over the duration of the study period (MolinariJobin et al. 2012). Additionally, the use of camera trapping has increased over time, leading to an increasing amount of $\mathrm{C} 1$ detections and therefore diminishing the proportion of $\mathrm{C} 3$ in the datasets (Molinari-Jobin et al. 2017). The learning process of citizens in scientific monitoring 
programs has been studied in the past (Dickinson et al. 2012; Jordan et al. 2012) and it was

found that the general public not only learned through participation but also became more aware of the general ecological issues and became more prone to understand scientific research (Bonney et al. 2009). We found that the probability to make a true detection was similar to the probability to detect the species in MU fitted by Molinari-Jobin et al. (2017). This makes sense because the probability to detect the species in MU is equal to the probability to make a true detection multiplied by the probability to classify a detection as unambiguous. We also found that there was a probability of 0.8 to classify a true detection as unambiguous. This may be due to the fact that observers in the network are highly competent at detecting the species and produce reliable data. This could also reflect that it is relatively easy to identify the signs of presence of lynx because there is almost no confusion possible with other species present in the area. Whenever the focus species can be mistaken for another one, if data quality is not sufficient (e.g. tracks in the snow for wolves which can be mistaken for dogs), true detections can be classified as ambiguous. There can also be false positive detections coming from misidentification when $b$ is low. In this case, the amount of true detections in ambiguous data will be non-negligible. In a case where $b$ is low and only unambiguous data is used, a large part of true presences can be missed and the resulting distribution will be underestimated (Miller et al. 2011).

The occupancy estimates under both models agree to suggest that the lynx case study corresponds to the LH simulation scenario (compare Table 1 to Appendix S4). When inspecting the distribution maps produced by MUA, we saw that adding ambiguous detections brought new and useful information. Some sites were estimated as occupied by MUA, while these same sites were estimated as non-occupied by MU (Fig 1). Because of the low occupancy of the lynx and its elusive behavior, the number of times the species was detected was very low. Because the probability to classify a detection as unambiguous $b$ was high, 
only few true detections were classified as ambiguous, which might explain why adding them did not change the parameter estimates but helped producing more precise estimates. In turn, it provides new insights in the context of managing a protected species (Guillera-Arroita et al. 2015). The sites we found to be occupied thanks to the incorporation of ambiguous detections could likely represent areas where the species is currently expanding. These same sites also point to places where lynx have not occurred before and negative interactions might occur due to the novelty of lynx presence. Sites that appeared occupied after including ambiguous data can inform the prediction of location of potential conflicts. Finally, if the objective is mapping the colonization front to, e.g., mitigate conflicts, ambiguous data should be included.

\section{Recommendations}

Dynamic occupancy models in general provide a powerful and natural analytical framework for changing species distributions (Kéry et al. 2013). More specifically, dynamic occupancy models accounting for misidentification represent a powerful method to deal with detections that cannot be categorized as certain in species distribution modeling. We recommend careful categorization of field observations into unambiguous or ambiguous detections, for instance by using several experts to classify the detections and use a standardized filtering classification process, to avoid false positive detections mistakenly classified as reliable data. This filtering process also allows avoiding too many detections that cannot be verified by rejecting some of them. If some detections cannot be checked by experts for instance and cannot be classified as unambiguous, observers might need to visit the sites where these detections were made to get more reliable detections. Even though occupancy models can deal with ambiguity, efforts should be put in the survey design and data collection to avoid the production of false positive detections or at least reduce their proportion. In the case of 
analyzing data from citizen-science, models accounting for false-positive detections can be a good tool to assess species distribution if a classification of detections is made (e.g.: unambiguous $v s$ ambiguous). In the case of a species occurring at low density such as the Eurasian lynx, additional information can bring new insights into the species distribution and help targeting specific sites where the species is likely to occur in the future.

\section{References}

Bailey, L.L., Mackenzie, D.I. \& Nichols, J.D. (2014) Advances and applications of occupancy models. Methods in Ecology and Evolution, 5, 1269-1279.

Bonney, R., Cooper, C.B., Dickinson, J., Kelling, S., Phillips, T., Rosenberg, K. V \& Shirk, J. (2009) Citizen Science : A Developing Tool for Expanding Science Knowledge and Scientific Literacy. 59, 977-984.

Chambert, T.C., Miller, D.A.W. \& Nichols, J.D. (2015) Modeling false positive detections in species occurrence data under different study designs. Ecology, 96, 332-339.

Chapron, G., Kaczensky, P., Linnell, J.D.C., von Arx, M., Huber, D., Andren, H., Lopez-Bao, J. V., et al. (2014) Recovery of large carnivores in Europe's modern human-dominated landscapes. Science, 346, 1517-1519.

Dickinson, J.L., Shirk, J., Bonter, D., Bonney, R., Crain, R.L., Martin, J., Phillips, T., et al. (2012) The current state of citizen science as a tool for ecological research and public engagement. Frontiers in Ecology and the Environment, 10, 291-297.

Elith, J. \& Leathwick, J.R. (2009) Species Distribution Models : Ecological Explanation and Prediction Across Space and Time. Annual review of ecology, evolution, and systematics, 40, 677-697. 
Eriksson, T. \& Dalerum, F. (2018) Identifying potential areas for an expanding wolf population in Sweden. Biological Conservation, 220, 170-181.

Fitzpatrick, M.C., Preisser, E.L., Ellison, A.M. \& Elkinton, J.S. (2009) Observer Bias and the Detection of Low-Density Populations. Ecological Applications, 19, 1673-1679.

Guillera-Arroita, G. (2017) Modelling of species distributions, range dynamics and communities under imperfect detection: advances, challenges and opportunities. Ecography, 40, 281-295.

Guillera-arroita, G., Lahoz-monfort, J.J., Elith, J., Gordon, A., Kujala, H., Lentini, P.E., Mccarthy, M.A., et al. (2015) Is my species distribution model fit for purpose ? Matching data and models to applications. Global Ecology and Biogeography, 24, 276-292.

Guisan, A. \& Thuiller, W. (2005) Predicting species distribution : offering more than simple habitat models. Ecology Letters, 8, 993-1009.

Isaac, N.J.B., Strien, A.J. Van, August, T.A., Zeeuw, M.P. De \& Roy, D.B. (2014) Statistics for citizen science : extracting signals of change from noisy ecological data. Methods in Ecology and Evolution, 5, 1052-1060.

IUCN (2012) IUCN Red List Categories and Criteria: version 3.1, 2nd edn. Gland, Switzerland and Cambridge, UK.

Jedrzejewski, W., Robinson, H.S., Abarca, M., Zeller, K.A., Velasquez, G., Paemelaere, E.A.D., Goldberg, J.F., et al. (2018) Estimating large carnivore populations at global scale based on spatial predictions of density and distribution - Application to the jaguar ( Panthera onca ). PLoS ONE, 1-25.

Jordan, R.C., Ballard, H.L. \& Phillips, T.B. (2012) Key issues and new approaches for evaluating citizen-science learning outcomes. Frontiers in Ecology and the Environment, 
10, 307-309.

443

444

445

446

447

448

449

450

451

452

453

454

455

456

457

458

459

460

461

462

463

464

Kéry, M., Guillera-arroita, G. \& Lahoz-monfort, J.J. (2013) Analysing and mapping species range dynamics using occupancy models. Journal of Biogeography, 40, 1463-1474.

Kosmala, M., Wiggins, A., Swanson, A. \& Simmons, B. (2016) Assessing data quality in citizen science. Frontiers in Ecology and the Environment, 14.

Louvrier, J., Duchamp, C., Lauret, V., Marboutin, E., Cubaynes, S., Choquet, R., Miquel, C., et al. (2018) Mapping and explaining wolf recolonization in France using dynamic occupancy models and opportunistic data. Ecography, 41, 647-660.

Mackenzie, D.I., Nichols, J.D., Hines, J.E., Knutson, M.G. \& Franklin, A.B. (2003) Estimating Site Occupancy, Colonization, and Local Extinction When a Species Is Detected Imperfectly. Ecology, 84, 2200-2207.

Marucco, F. \& Mcintire, E.J.B. (2010) Predicting spatio-temporal recolonization of large carnivore populations and livestock depredation risk : wolves in the Italian Alps. Journal of Applied Ecology, 47, 789-798.

Mcclintock, B.T., Bailey, L.L., Pollock, K.H. \& Simons, T.R. (2010) Unmodeled observation error induces bias when inferring patterns and dynamics of species occurrence via aural detections. Ecology, 91, 2446-2454.

Mckinley, D.C., Miller-rushing, A.J., Ballard, H.L., Bonney, R., Brown, H., Cook-patton, S.C., Evans, D.M., et al. (2017) Citizen science can improve conservation science, natural resource management, and environmental protection. Biological Conservation, 208, 15-28.

Miller, D.A., Nichols, J.D., McClintock, B.T., Grant, E.H.C., Bailey, L.L. \& Weir, L.A. (2011) Improving occupancy estimation when two types of observational error occur: 
466

467

468

469

470

471

472

473

474

475

476

477

478

479

480

481

482

483

484

485

Miller, D.A.W., Nichols, J.D., Gude, J.A., Rich, L.N., Podruzny, K.M., Hines, J.E. \& Mitchell, M.S. (2013) Determining Occurrence Dynamics when False Positives Occur: Estimating the Range Dynamics of Wolves from Public Survey Data. PLoS ONE, 8.

Molinari-Jobin, A., Kéry, M., Marboutin, E., Marucco, F., Zimmermann, F., Molinari, P., Frick, H., et al. (2017) Mapping range dynamics from opportunistic data: Spatiotemporal modelling of the lynx distribution in the Alps over 21 years. Animal Conservation, 1-13.

Molinari-Jobin, A., Kéry, M., Marboutin, E., Molinari, P., Koren, I., Fuxjäger, C., Breitenmoser-Würsten, C., et al. (2012) Monitoring in the presence of species misidentification: The case of the Eurasian lynx in the Alps. Animal Conservation, 15, $266-273$.

Ripple, W.J., Beschta, R.L., Fortin, J.K. \& Robbins, C.T. (2014) Trophic cascades from wolves to grizzly bears in Yellowstone. Journal of Animal Ecology, 83, 223-233.

Royle, J.A. \& Kéry, M. (2007) A Bayesian state-space formulation of dynamic occupancy models. Ecology, 88, 1813-1823.

Royle, J.A. \& Link, A.W. (2006) Generalized site occupancy models allowing for false positive and false negative errors. Ecology, 87, 835-841.

Silvertown, J. (2009) A new dawn for citizen science. Trends in Ecology and Evolution, 24, $467-471$. 
Table 1: Parameters values for the simulation scenarios

\begin{tabular}{lllll}
\hline Scenarios & Initial occupancy & Colonization & Probability to classify & False positive \\
probability $\psi_{1}$ & probability $\gamma$ & a true detection as & detection \\
& & & unambiguous $b$ & probability $p_{10}$
\end{tabular}

\begin{tabular}{|c|c|c|c|c|}
\hline \multirow[t]{2}{*}{ "high" occupancy } & 0.8 & 0.4 & 0.8 & 0.01 \\
\hline & & & & 0.5 \\
\hline \multirow[t]{5}{*}{ "high" $b(\mathrm{HH})$} & & & & 0.10 \\
\hline & & & & 0.15 \\
\hline & & & & 0.20 \\
\hline & & & & 0.25 \\
\hline & & & & 0.30 \\
\hline \multirow[t]{2}{*}{ "high" occupancy } & 0.8 & 0.4 & 0.5 & 0.01 \\
\hline & & & & 0.5 \\
\hline \multirow[t]{5}{*}{ "low" $b$ (HL) } & & & & 0.10 \\
\hline & & & & 0.15 \\
\hline & & & & 0.20 \\
\hline & & & & 0.25 \\
\hline & & & & 0.30 \\
\hline \multirow[t]{2}{*}{ "low" occupancy } & 0.1 & 0.1 & 0.8 & 0.01 \\
\hline & & & & 0.5 \\
\hline \multirow[t]{5}{*}{ "high" $b(\mathrm{LH})$} & & & & 0.10 \\
\hline & & & & 0.15 \\
\hline & & & & 0.20 \\
\hline & & & & 0.25 \\
\hline & & & & 0.30 \\
\hline \multirow[t]{2}{*}{ "low" occupancy } & 0.1 & 0.1 & 0.5 & 0.01 \\
\hline & & & & 0.5 \\
\hline \multirow[t]{5}{*}{ "low" $b(\mathrm{LL})$} & & & & 0.10 \\
\hline & & & & 0.15 \\
\hline & & & & 0.20 \\
\hline & & & & 0.25 \\
\hline & & & & 0.30 \\
\hline
\end{tabular}

487

488

489

490 
Table 2: Parameters estimates for the detection probabilities from both dynamic occupancy models accounting for unambiguous data only and accounting for unambiguous and ambiguous data; the first column corresponds to the parameters estimates for the detection probability from the model with unambiguous data only, the second column correspond to the parameters estimates for the probability of correctly detecting the species given a site is occupied from the dynamic occupancy model accounting for unambiguous and ambiguous data, the last columns correspond to the parameters estimates for the probability of incorrectly detecting the species given a site is unoccupied; posterior means, standard deviation and the lower and upper bound of the $95 \%$ Bayesian credible interval are given. Effects with 95\% Bayesian credible intervals that do not contain zero are in bold. 


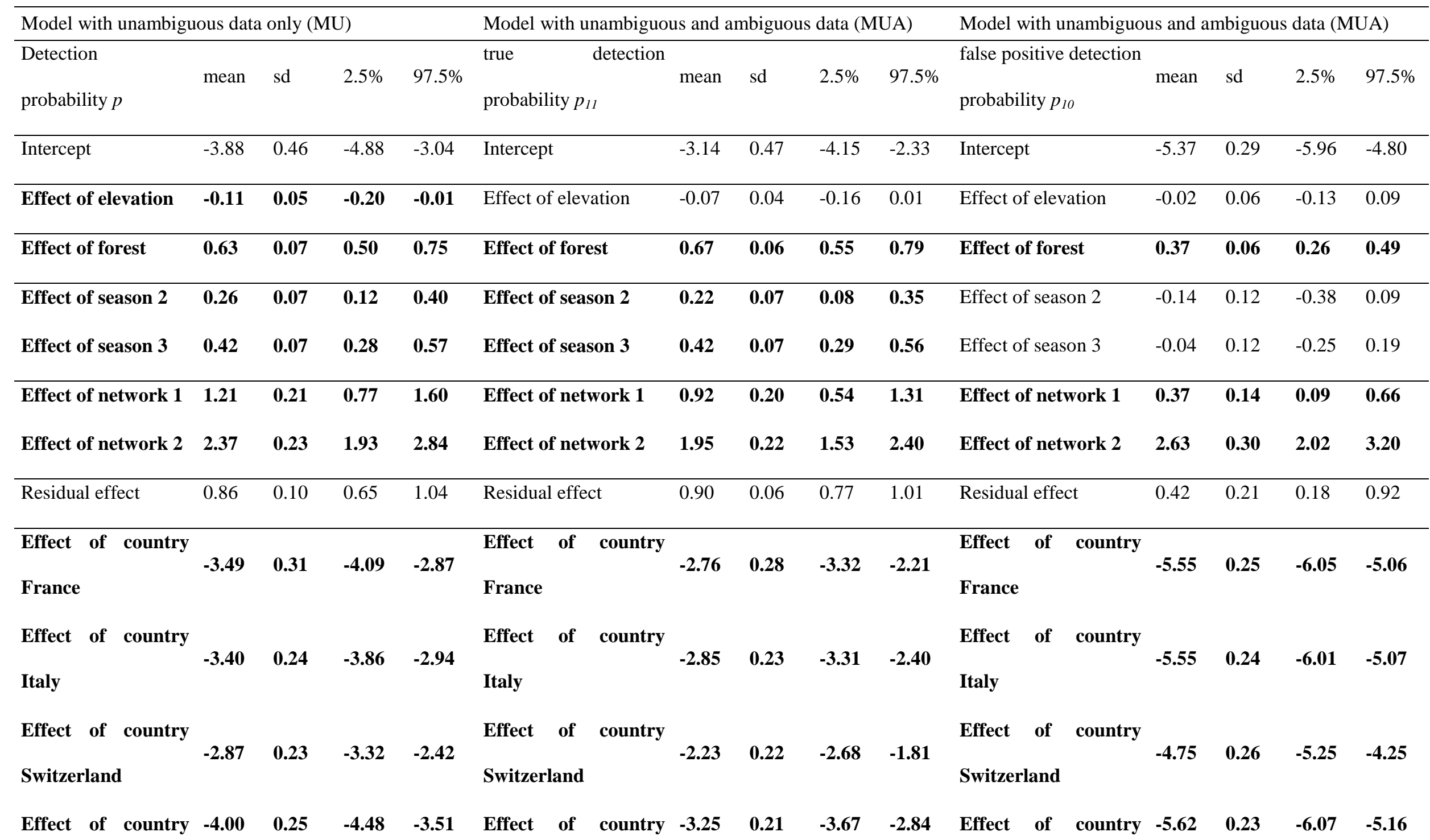




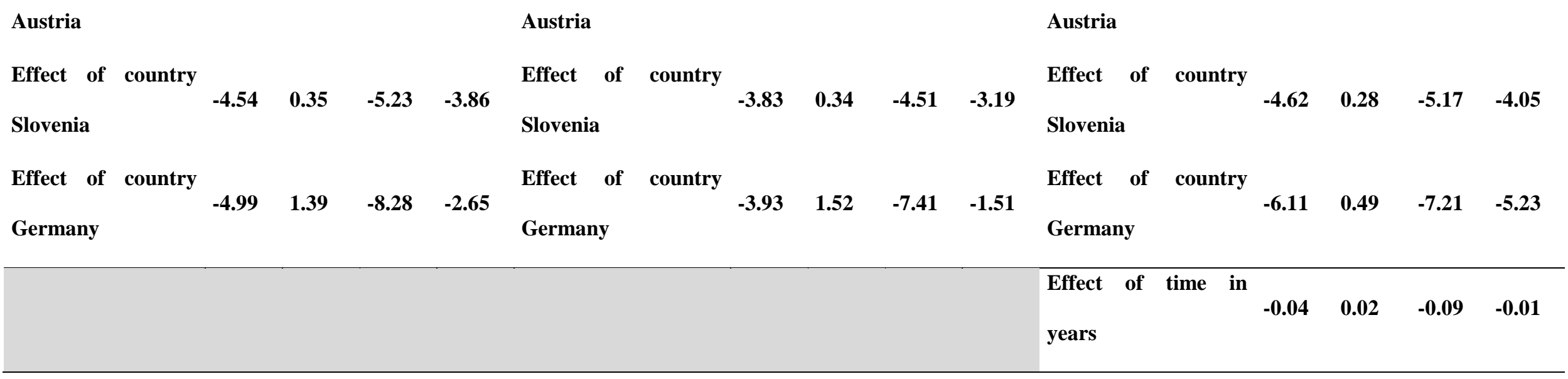


500

501

502 